\title{
A case of conversion disorder showing transient hemiplegia after general anesthesia
}

\author{
Chieko Nakagawa Yoshito Shiraishi · \\ Shigehito Sato
}

Received: 24 May 2009/Accepted: 8 January 2010/Published online: 11 March 2010

(C) Japanese Society of Anesthesiologists 2010

To the Editor:

Hemiplegia due to conversion disorder is a diagnosis of exclusion, only to be considered after more serious diseases have been eliminated. We experienced a patient who complained of an inability to move his left extremities and showed no response to painful stimuli after emerging from general anesthesia.

The patient was a 22-year-old Chinese male with American Society of Anesthesiologists (ASA) physical status I who was scheduled for removal of the oral plate in the maxilla under general anesthesia. Although he spoke only limited Japanese, informed consent was obtained. General anesthesia was induced and maintained without any notable events. Following the surgery, the patient was completely alert—but unable to move his left arm and leg, which also did not respond to painful stimuli. Neurological tests revealed paralysis of the left side extremities and the disappearance of perception in these regions. The results of other neurological examinations were normal, and radiological tests did not show abnormalities; however, we initiated treatment for a brain infarction. Twelve hours after the start of treatment, the patient suddenly moved his left extremities, and $24 \mathrm{~h}$ after the operation his recovery was complete. The psychiatrist diagnosed hemiplegia due to conversion disorder.

C. Nakagawa $(\varangle) \cdot$ Y. Shiraishi

Surgical Center, Hamamatsu University Hospital,

1-20-1 Handayama, Higashi-ku, Hamamatsu,

Shizuoka 431-3192, Japan

e-mail: cnaka@hama-med.ac.jp

S. Sato

Department of Anesthesiology and Intensive Care,

Hamamatsu University School of Medicine, Hamamatsu, Japan
Conversion disorder is diagnosed when there are one or more "unexplained" physical symptoms involving the musculature and sensory system, such as paradoxical paralyses, seizures, sensory deficits, and pain [1]. Some patients show signs of immaturity, histrionic behavior, sexualization of relationships, low frustration tolerance, shallow interpersonal ties, and dependency [2]. Conversion disorder is interpreted as physical symptoms that arise from unconscious conflicts in patients during an adaptation to difficult situations. We speculate that the conversion disorder in our patient may have been caused by the need to undergo surgery in a foreign country where his inability to communicate sufficiently with physicians and medical staff may have caused excessive stress. Our experience suggests that conversion disorder should be considered in cases with unexplainable physical symptoms after all other possible causes have been eliminated, especially those requiring prompt intervention. When physicians consider the symptom to be due to conversion disorder, psychiatrists should be consulted and therapies initiated as soon as possible.

\section{References}

1. Miyaoka Y. Somatoform disorders (in Japanese). Rinshou Seishin Igaku (Jpn J Clin Psychiatry). 2006;35:163-7.

2. Adams AP, Goroszeniuk T. Hysteria a cause of failure to recover after anesthesia. Anaesthesia. 1991;46:932-4. 